\title{
SU(2) meets SU(3) in lattice-Landau-gauge gluon and ghost propagators
}

\section{A. Cucchieri}

Instituto de Física de São Carlos, Universidade de São Paulo,

Caixa Postal 369, 13560-970 São Carlos, SP, Brazil E-mail: attilio@ifsc.usp.br

\section{T. Mendes}

Instituto de Física de São Carlos, Universidade de São Paulo,

Caixa Postal 369, 13560-970 São Carlos, SP, Brazil E-mail: mendes@ifsc . usp.br

\section{Orlando Oliveira*}

Department of Physics, University of Coimbra, 3004516 Coimbra, Portugal

E-mail: orlandodteor.fis.uc.pt

\section{P. J. Silva}

Department of Physics, University of Coimbra, 3004516 Coimbra, Portugal

E-mail: psilva@teor.fis.uc.pt

A comparative study of the lattice Landau gauge gluon and ghost propagators for SU(2) and SU(3) pure Yang-Mills theories is carried out. The data were specially produced with equivalent lattice parameters to allow for a careful comparison of the two cases. We find very good agreement between the two theories. Our results seem to confirm the predicton of Schwinger-Dyson equations that the infrared exponents are independent of the gauge group $\mathrm{SU}(\mathrm{N})$.

The XXV International Symposium on Lattice Field Theory

July 30 - August 42007

Regensburg, Germany

\footnotetext{
${ }^{*}$ Speaker.
} 


\section{Introduction and Motivation}

The investigation of the infrared limit of QCD is of central importance for the comprehension of the mechanisms of quark and gluon confinement and of chiral-symmetry breaking. However, despite the recent progress, we still do not have the full picture of the infrared structure of YangMills theories.

In what concerns gluon confinement, in Landau gauge, the infrared behavior of gluon and ghost propagators is linked with the Gribov-Zwanziger [1,2] and the Kugo-Ojima [3] confinement scenarios. These confinement mechanisms predict, at small momenta, an enhanced ghost propagator and a suppression of the gluon propagator. Analytic studies of gluon and ghost propagators using Schwinger-Dyson equations (SDE) $[4,5,6]$ seem to agree with the above scenarios. Schwinger-Dyson equations are an infinite tower of nonlinear equations. Typically, the computation of a solution requires the definition of a truncation scheme and the parametrization of vertices. The above mentioned solutions are not the only known solutions. Indeed, in $[7,8]$ the authors found a set of solutions which do not comply with the above mechanisms. In what concerns the lattice results for the gluon and ghost propagators, in Landau gauge, one side they seem to support the analytical studies $[9,10,11]$, on the other side they do not confirm the precise predictions obtained with SDE [12]. The solution of this apparent puzzle requires further studies.

In the Schwinger-Dyson equations, when dynamic quarks are neglected, assuming that $g^{2} \sim$ $1 / N_{c}$ — as suggested by analysis of the large $N_{c}$ limit [13] — the SDE predict that gluon and ghost propagators are independent of the number of colors (in the nonperturbative regime). In particular, they predict for the gluon and for the ghost propagators an infrared exponent that is independent of the gauge group $S U\left(N_{c}\right)$. In this paper, we carry out a comparative study of lattice Landau gauge propagators for these two gauge groups. Our data were especially produced by considering equivalent lattice parameters in order to allow a careful comparison of the two cases. For details on the simulation see [14]. For another study comparing $S U(2)$ and $S U(3)$ propagators see [15]. In the following the effect of Gribov copies is not taken into account.

\section{Numerical Simulations}

We consider four different sets of lattice parameters, with the same lattice size $N^{4}$ and the same physical lattice spacing $a$ for the two gauge groups (see Table 1). The first three cases are chosen to yield approximately the same physical lattice volume $V \approx(1.7 \mathrm{fm})^{4}$. This allows a comparison of discretization effects. The fourth case corresponds to a significantly larger physical volume, $V \approx(3.2 \mathrm{fm})^{4}$, in order to study finite-size effects. For all four cases, 50 configurations were generated using the Wilson action.The gluon and the ghost propagators

$$
\begin{aligned}
D_{\mu v}^{a b}\left(k^{2}\right) & =\delta^{a b}\left(\delta_{\mu v}-\frac{k_{\mu} k_{v}}{k^{2}}\right) D\left(k^{2}\right), \\
G^{a b}\left(k^{2}\right) & =-\delta^{a b} G\left(k^{2}\right)
\end{aligned}
$$

were computed for four different types of momenta: $(k, 0,0,0),(k, k, 0,0),(k, k, k, 0)$ and $(k, k, k, k)$. In the computation of $D\left(k^{2}\right)$ and $G\left(k^{2}\right)$, an average over equivalent momenta and color components was always performed. 


\begin{tabular}{lllll}
$N^{4}$ & $a(\mathrm{fm})$ & $N a(\mathrm{fm})$ & $\beta_{S U(2)}$ & $\beta_{S U(3)}$ \\
\hline $16^{4}$ & 0.102 & 1.632 & 2.4469 & 6.0 \\
$24^{4}$ & 0.073 & 1.752 & 2.5501 & 6.2 \\
$32^{4}$ & 0.054 & 1.728 & 2.6408 & 6.4 \\
$32^{4}$ & 0.102 & 3.264 & 2.4469 & 6.0 \\
\hline
\end{tabular}

Table 1: Lattice setup. The lattice spacing was computed from the string tension, assuming $\sqrt{\sigma}=440$ $\mathrm{MeV}$.
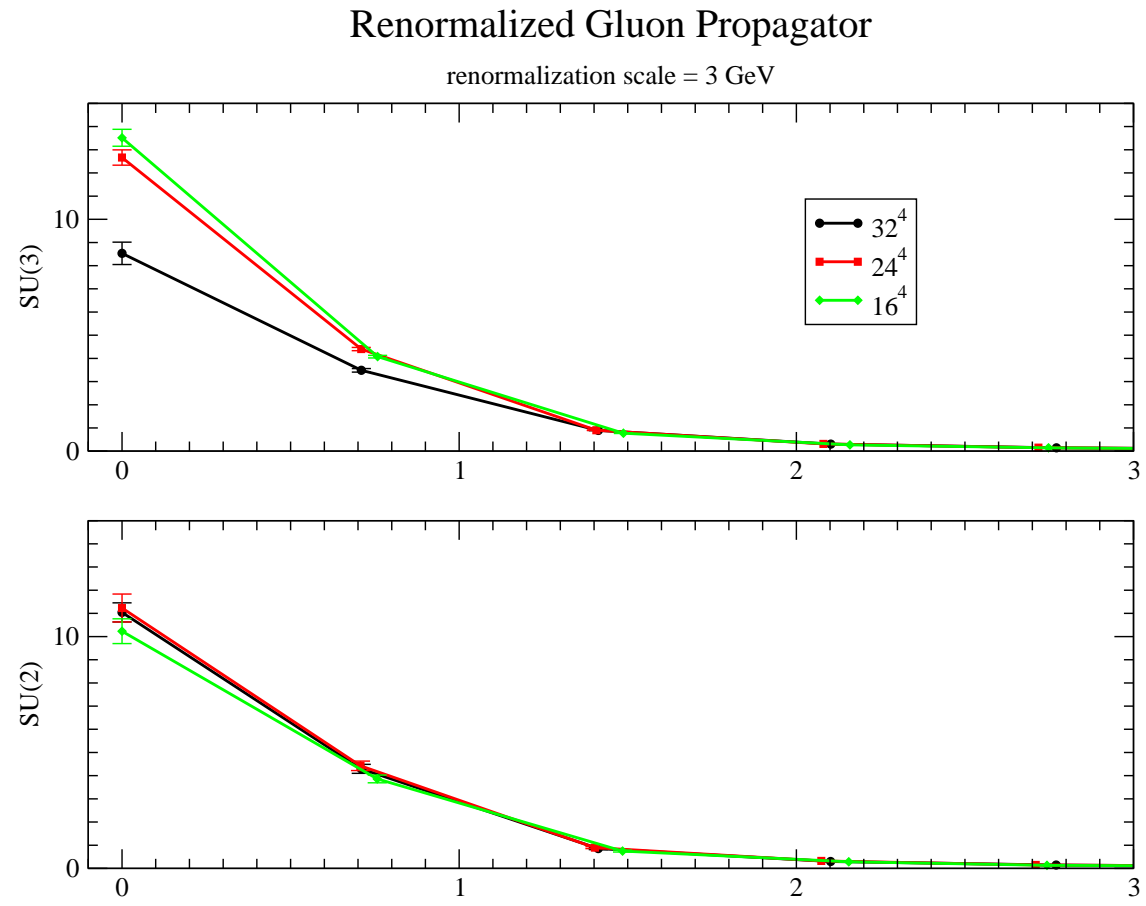

Figure 1: Gluon propagator as function of momenta given in $\mathrm{GeV}$ for lattices with volume $V \approx(1.7 \mathrm{fm})^{4}$.

In order to compare the propagators from the different simulations, the gluon and ghost propagators were renormalized accordingly to

$$
\left.D\left(q^{2}\right)\right|_{q^{2}=\mu^{2}}=\frac{1}{\mu^{2}},\left.\quad G\left(q^{2}\right)\right|_{q^{2}=\mu^{2}}=\frac{1}{\mu^{2}},
$$

using $\mu=3 \mathrm{GeV}$ as a renormalization point. The lattice data were interpolated (using splines) to allow the use of such a renormalization point in all the simulations.

\section{The Propagators}

The gluon propagator for $V \approx(1.7 \mathrm{fm})^{4}$ is reported in figure 1 . In figure 2 , the data for different volumes, same $\beta$ value is displayed. The corresponding figures for the ghost propagator are fig. 3 and fig. 4, respectively. 

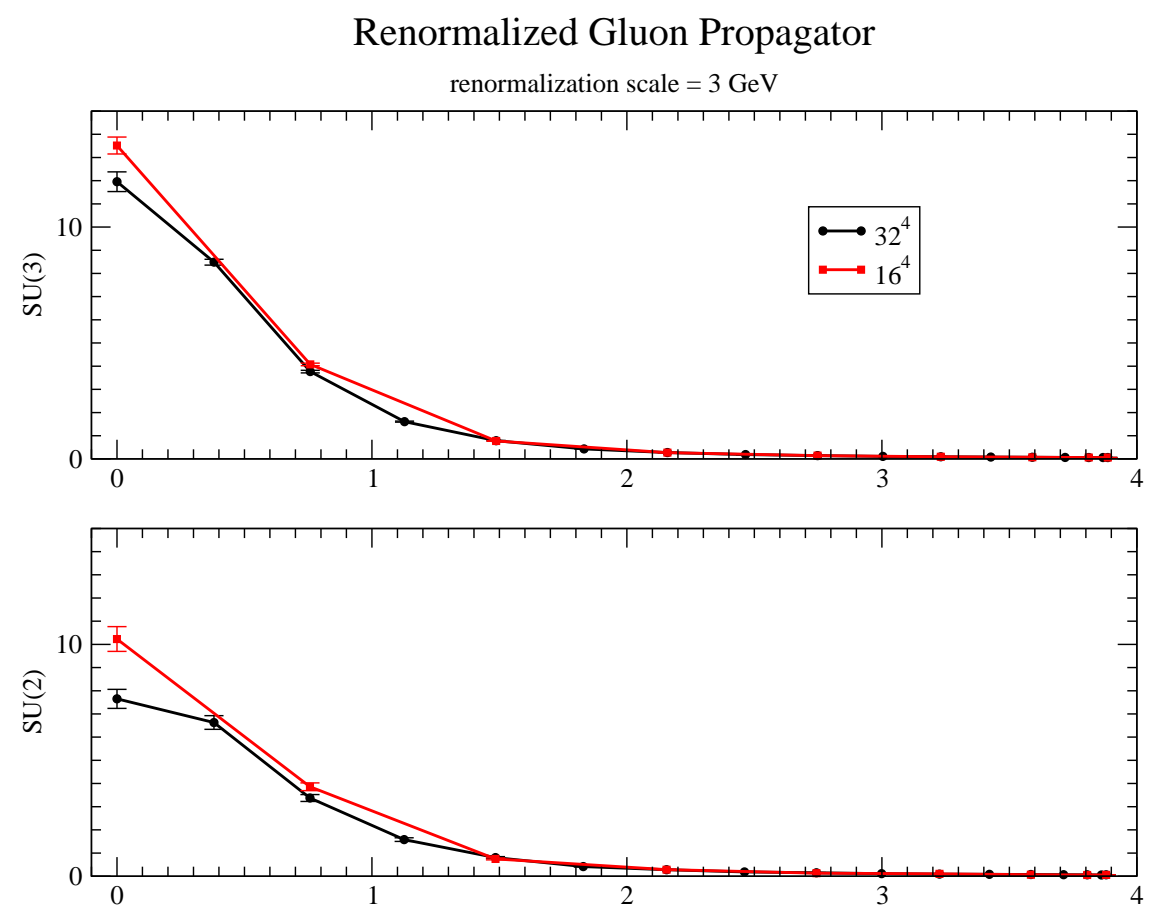

Figure 2: Gluon propagator as function of momenta given in $\mathrm{GeV}$ for $\beta_{S U(3)}=6.0$ and $\beta_{S U(2)}=2.4469$.

For the gluon propagator, figure 1 show some discretization effects which are stronger for the SU(3) data. On the other hand, figure 2 shows finite volume effects, specially for SU(2). In order to try to understand such effects, in figure 5 one plots the ratios of $\mathrm{SU}(3)$ over $\mathrm{SU}(2)$ propagators for all the simulations. Note that the plots include ratios of $D(0)$, i.e. the most left point should be taken with care. In what concerns the gluon propagator, given the relatively small statistics and given that there is no clear systematics in data, one can not conclude on the nature of observed small differences. Anyway, the SU(3) and SU(2) propagators are, at least, qualitatively similar. Given the small differences one can also claim quantitative agreement between the two propagators.

In what concerns the ghost propagator, the data seems more stable than the gluon points. Indeed, comparing figures 1-4 and the ratios of propagators in fig. 5, fig 6 the ghost data fluctuates less. Moreover, for the full range of momenta the ratios of ghost propagators are compatible with one at the level of two standard deviations. Therefore, for the ghost propagator one can conclude in favour of quantitative and qualitative agreement between $\mathrm{SU}(2)$ and $\mathrm{SU}(3)$.

\section{Results and Conclusions}

In summary, considering a careful choice of the lattice parameters, we were able to carry out an unambiguous comparison of the lattice Landau gluon and ghost propagators for $S U(2)$ and $S U(3)$ gauge theories. The data show that the two cases have very similar finite-size and discretization effects. Moreover, we find very good agreement between the two Yang-Mills theories (for our values of momenta larger than $1 \mathrm{GeV}$ ), for all lattice parameters and for all types of momenta. Below $1 \mathrm{GeV}$, the results for the two gauge groups show some differences, especially for the gluon 


\section{Renormalized Ghost Propagator}
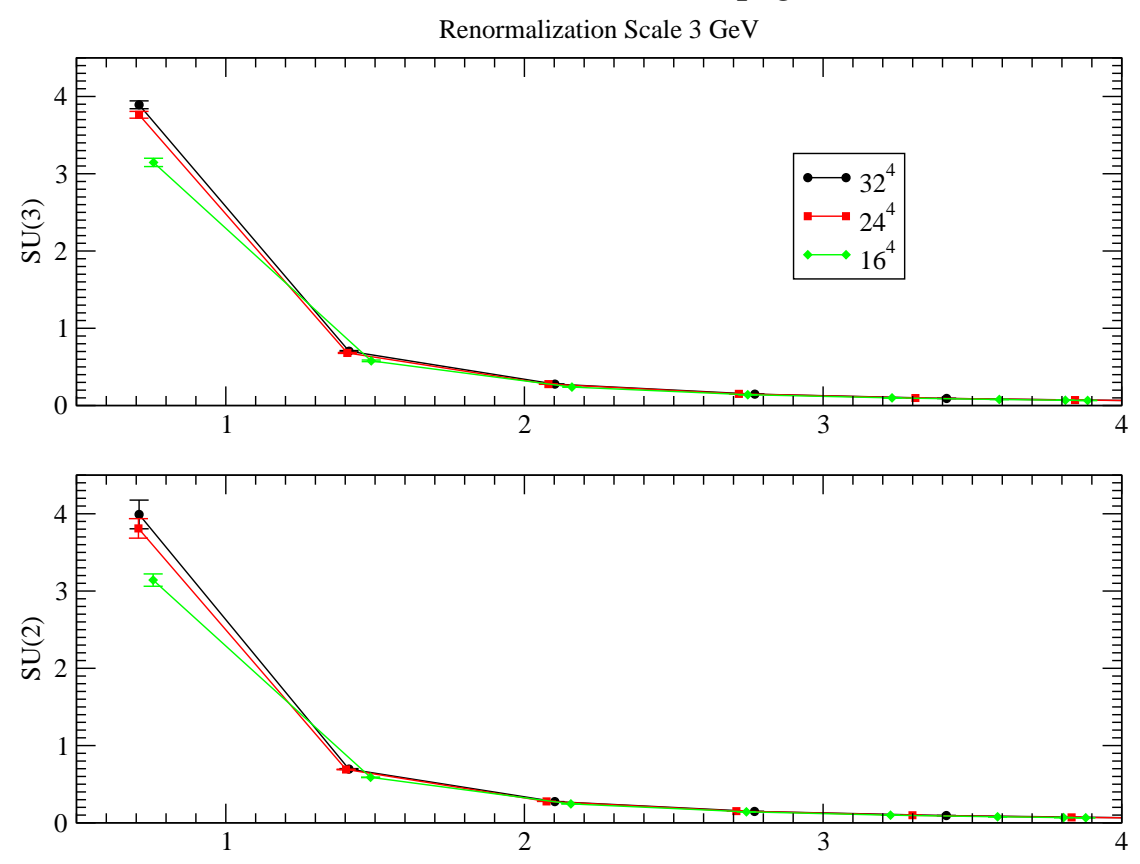

Figure 3: Ghost propagator as function of momenta given in $\mathrm{GeV}$ for lattices with volume $V \approx(1.7 \mathrm{fm})^{4}$.

\section{Renormalized Ghost Propagator}
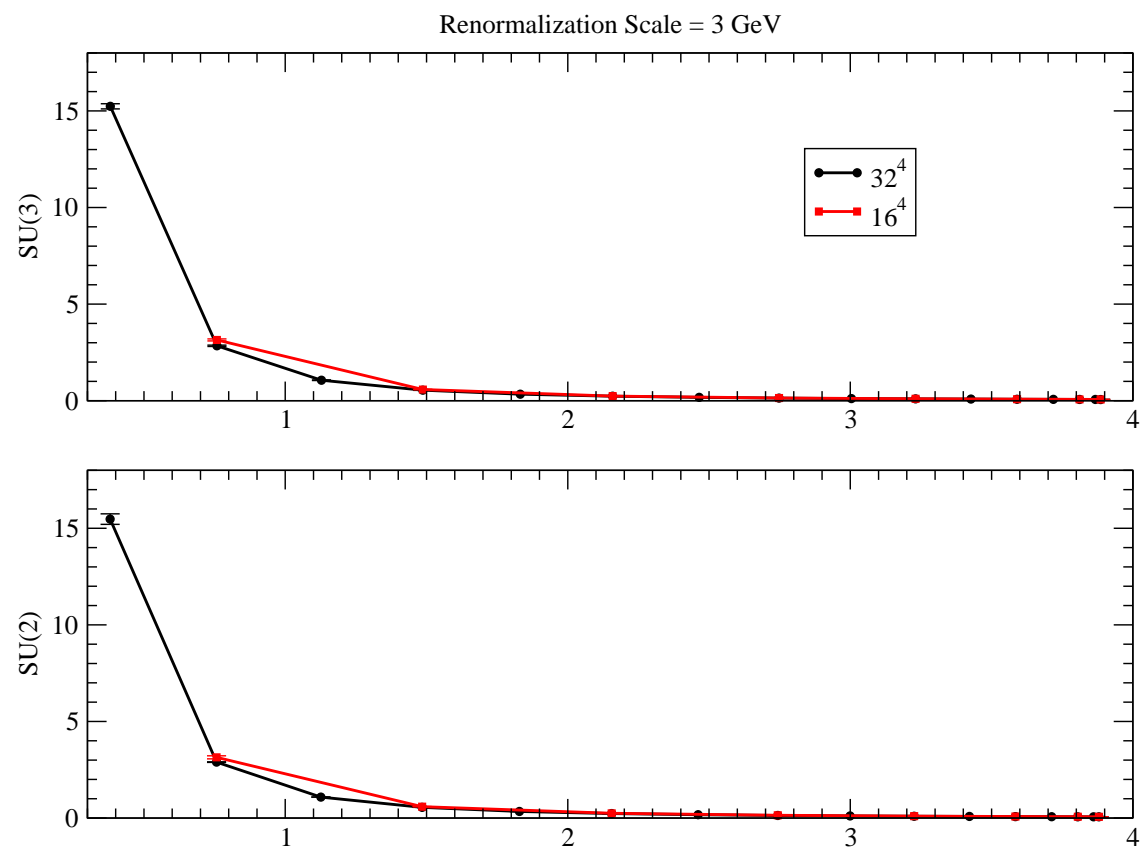

Figure 4: Ghost propagator as function of momenta given in $\mathrm{GeV}$ for $\beta_{S U(3)}=6.0$ and $\beta_{S U(2)}=2.4469$. 


\section{Ratios of Gluon SU(3)/SU(2) Propagator}

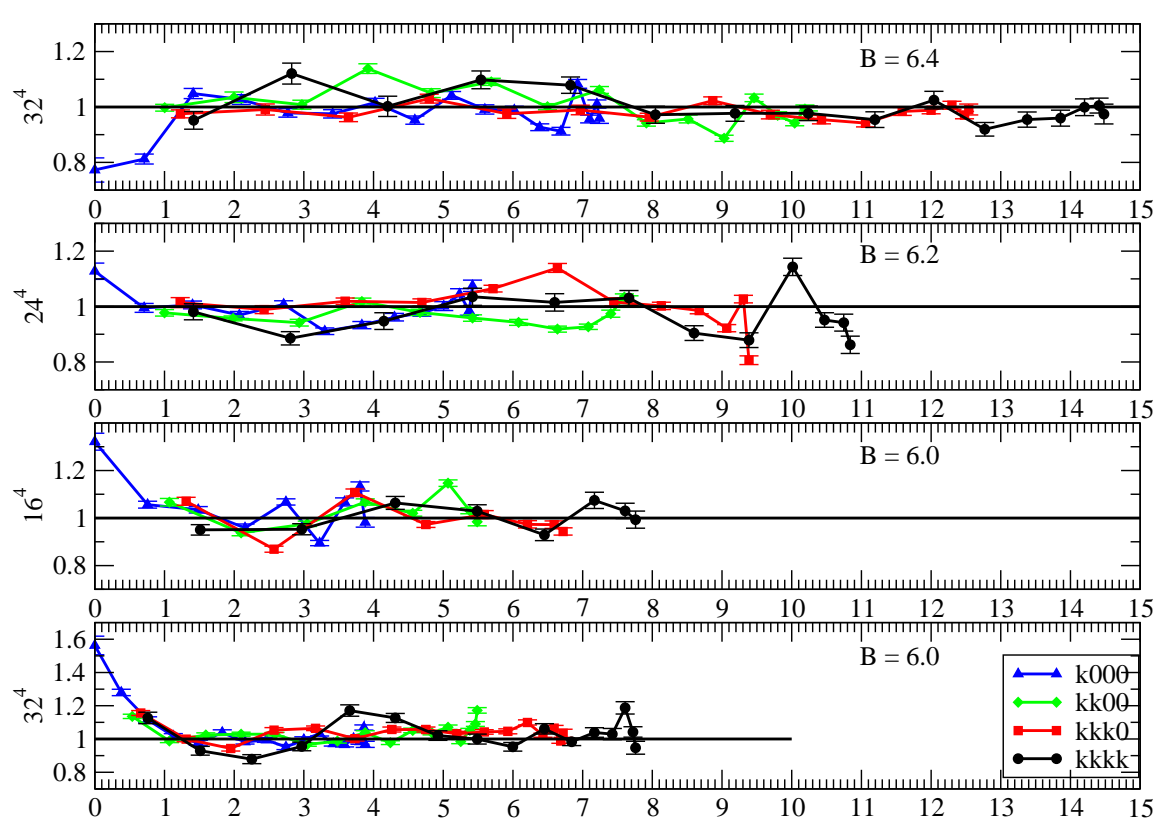

Figure 5: $\mathrm{SU}(3) / \mathrm{SU}(2)$ gluon propagator as function of momenta given in GeV.

\section{Ratios of Ghost SU(3)/SU(2) Propagators}

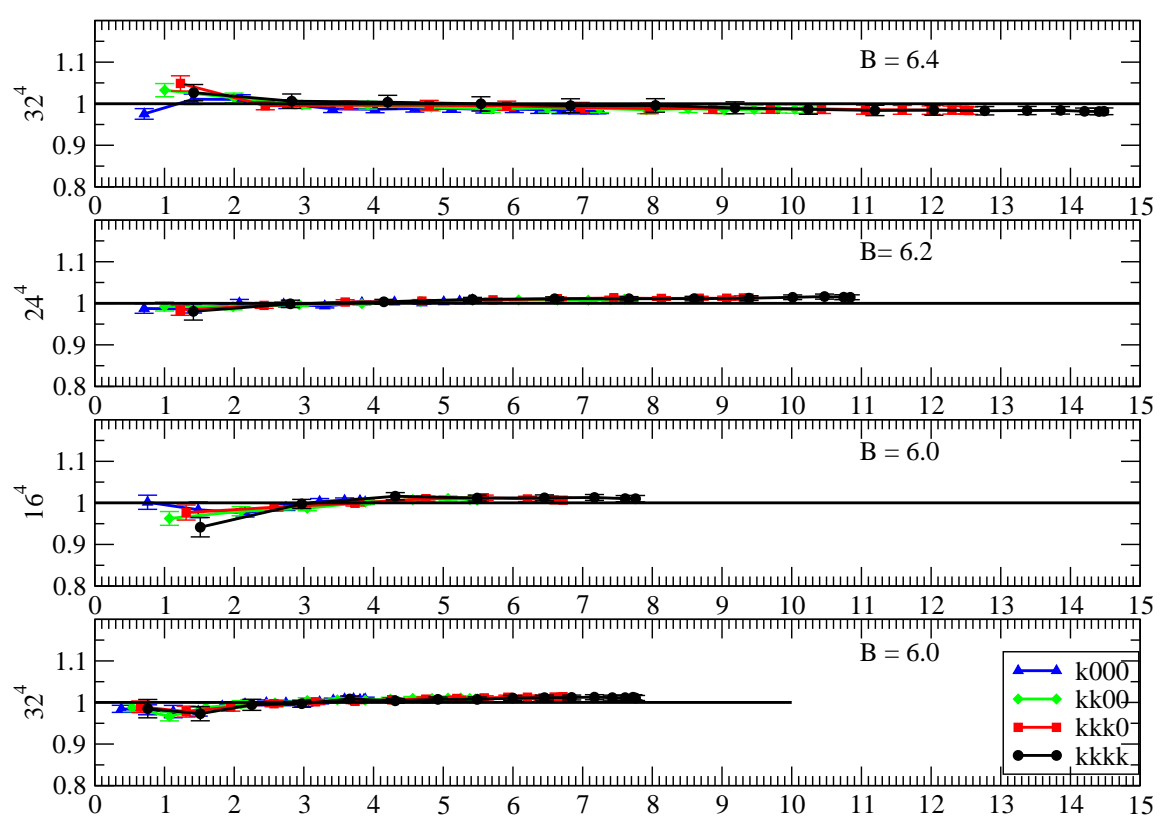

Figure 6: $\mathrm{SU}(3) / \mathrm{SU}(2)$ ghost propagator as function of momenta given in GeV. 
propagator. However, given the lattice volumes considered, further studies are required before drawing conclusions about the comparison between $S U(2)$ and $S U(3)$ propagators in the deep-IR region. In this sense, we claim that our results support the prediction from the Schwinger-Dyson equations that the propagators are the same for all $S U\left(N_{c}\right)$ groups in the nonperturbative region.

\section{Acknowledgments}

P.J.S.acknowledges F.C.T. financial support via grant SFRH/BD/10740/2002. This work was supported in part by F.C.T. under contracts POCI/FP/63436/2005 and POCI/FP/63923/2005.

\section{References}

[1] V. N. Gribov, Nucl. Phys. B139, 1 (1978).

[2] D. Zwanziger, Phys. Lett. B257, 168 (1991); Nucl. Phys. B364, 127 (1991); Nucl. Phys. B412, 657 (1994).

[3] T. Kugo, I. Ojima, Prog. Theor. Phys. Suppl. 66, 1 (1979) [Erratum Prog. Theor. Phys. Suppl. 71, 1121 (1984)].

[4] L. von Smekal, A. Hauck, R. Alkofer, Ann. Phys. 267, 1 (1998).

[5] C. Lerche, L. von Smekal, Phys. Rev. D65, 125006 (2002).

[6] D. Zwanziger, Phys. Rev. D65, 094039 (2002).

[7] A. C. Aguilar, A. A. Natale, P. S. Rodrigues da Silva, Phys. Rev. Lett. 90, 152001 (2003).

[8] A. C. Aguilar, A. A. Natale, JHEP 408, 57 (2004).

[9] O. Oliveira, P. J. Silva, arXiv:0705.0964[hep-lat], (2007).

[10] P. J. Silva, O. Oliveira, Phys. Rev.D74, 034513 (2006).

[11] P. J. Silva, O. Oliveira, PoS LATTICE2007, 323 (2007).

[12] A. Cucchieri, T. Mendes, $P o S($ LATTICE2007), 297 (2007).

[13] G. t'Hooft, Nucl. Phys. B72, 461 (1974).

[14] A. Cucchieri, T. Mendes, O. Oliveira, P. J. Silva, arXiv:0705.3367[hep-lat], (2007).

[15] A. Sternbeck, D.B. Leinweber, L. von Smekal, A.G. Williams, PoS(LATTICE2007), 340 (2007). 